\title{
SYNTHESIS AND CHARACTERIZATION OF N-CHLOROPICOLINAMIDE: A NEW, MILD, STABLE, EFFECTIVE AND EFFICIENT OXIDANT FOR ORGANIC SUBSTRATES
}

\author{
M. Subalakshmi* and V. Priya \\ Department of Chemistry, Holy Cross College/ Bharathidasan University, \\ Tiruchirappalli-2, TamilNadu, India. \\ E-mail: subachemist1994@gmail.com
}

\begin{abstract}
The new oxidant N-Chloropicolinamide (NCP) is synthesized by the chlorination of picolinamide using trichloroisocyanuric acid. The physical constant, formal redox potential, element analysis and spectra characterization (IR, UV, ${ }^{1} \mathrm{H}-\mathrm{NMR}, \mathrm{C}^{13}-\mathrm{NMR}$ and mass spectrum) confirms the presence of nitrogen-halogen bond. It is prepared by a simple method giving a high yield in a short period of time. It is found to be a mild and stable oxidant and formal redox potential of N-chloropicolinamide shows that it can be used as an effective source of positive halogen.
\end{abstract}

Keywords: N-Chloropicolinamide, Oxidant, Kinetics, Mechanism. N-halo Compound.

(c) RASĀYAN. All rights reserved

\section{INTRODUCTION}

Chemical kinetics is a branch of chemistry that deals with the rate of reaction. The chemistry of N-halo compounds forms a separate branch which has large synthetic importance. ${ }^{1} \mathrm{~N}$-halo compounds have been widely employed as oxidizing and halogenating reagents in organic compounds. The electronegativity of halogens except fluorine is less than that of nitrogen. ${ }^{2-5}$ Hence they gain a positive charge when linked with nitrogen. ${ }^{6-8}$ The electronegativity of nitrogen is further enhanced by linking it with electronwithdrawing groups such as acyl groups. Thus N-halo compounds are given as positive halogen compounds. ${ }^{9} \mathrm{~N}$-halo compounds have been widely used as oxidants in kinetic studies for the oxidation of different substrates. ${ }^{10-11}$ N-halo reagents have been used as a catalyst in a variety of chemical transformation for the safeguard of functional groups. ${ }^{12}$

The present paper deals with the synthesis and characterization of N-Chloropicolinamide for the rapid oxidation of organic substrates. ${ }^{13}$ This oxidant offer advantages like a simple method of synthesis, low toxicity and mild nature with significant stability. ${ }^{14}$

\section{EXPERIMENTAL}

A simple method was developed for the preparation of $\mathrm{N}$-Chloropicolinamide (NCP). In a 250ml round bottom flask $3.932 \mathrm{~g}$ of nicotinamide was dissolved in methanol. Trichloroisocyanuric acid(TCICA), $1.371 \mathrm{~g}$ was added and cyanuric acid was precipitated. After stirring for 1 hour the mixture was vacuum filtred and the solid was washed with methylene chloride. Solvents were removed by using a rotary evaporator and the final residue of N-Chloropicolinamide (92\% yield) was obtained. Recrystallization of NCP was done with ether solvent. ${ }^{15}$
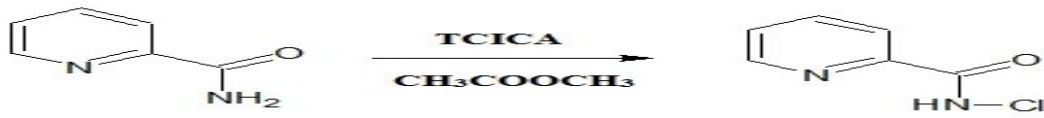

Fig.-1: Rection for the preparation of N-Chloropicolinamide

Rasayan J. Chem., 12(3), 1493-1495(2019)

http://dx.doi.org/10.31788/RJC.2019.1235213

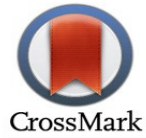


RASĀYAN J. Chem.

Vol. 12 | No. 3 |1493 - 1495| July - September | 2019

\section{RESULTS AND DISCUSSION}

Using thin-layer chromatography (TLC) purity of the N-Chloropicolinamide was checked by observing a single spot on TLC. The IR Spectrum of nicotinamide and NCP is given in Table-1.

Table-1

\begin{tabular}{c|c|c}
\hline Assignment & Picolinamide (PA) & N-Chloropicolinamide(NCP) \\
\hline $\mathrm{N}-\mathrm{H}$ (str.) $\mathrm{H}-$ bonded & 3180,3419 & 3438 \\
\hline $\mathrm{C}=\mathrm{O}$ (str.) (primary amide) & 1663 & 1673 \\
\hline $\mathrm{C}=\mathrm{O}$ (str.) (secondary amide) & - & - \\
\hline $\mathrm{NH}$ (def.) (primary amide) & 1604 & 822 \\
\hline $\mathrm{N}-\mathrm{Cl}$ (secondary amide) & - & 1251 \\
\hline $\begin{array}{c}\mathrm{C}-\mathrm{H} \text { (def.) } \text { of substituted } \\
\text { pyridine ring }\end{array}$ & 825 & \\
\hline
\end{tabular}

Pyridine ring protons in both picolinamide and NCP has shown same chemical shifts without any shifting, the proton peak corresponding to $\mathrm{NH}_{2}$ group in $\mathrm{NCP}$ is slightly shifted to a larger value while the integration of the peak was half. These shifts in $\mathrm{NH}$ proton shows that one proton of the $\mathrm{NH}_{2}$ group is substituted by the chlorine atom.

The UV spectrum of $\lambda_{\max }$ at 301 is responsible for the $\pi-\pi^{*}$ transition of PA. The frequency at 322 shows the shift in the peak due to the replacement of $\mathrm{H}$ by $\mathrm{Cl}$ in NCP.

${ }^{1} \mathrm{H}-\mathrm{NMR} \delta: 7.5$ ( due to $\mathrm{C}_{3}-\mathrm{H}$ and $\mathrm{C}_{5}-\mathrm{H}$ ), $\delta: 7.9$ (due to $\mathrm{C}_{4}-\mathrm{H}$ ) and $\delta: 8.6$ (due to $\mathrm{C}_{6}-\mathrm{H}$ ). The signal for the same N-Chloropicolinamide and the peak corresponding to $(-\mathrm{CONH})$ was slightly shifted to $\delta: 7.8$.

The ${ }^{13} \mathrm{C}$-NMR signal of PA and NCP was also recorded. The $\delta$ value of carbonyl carbon is $166.53 \mathrm{ppm}$ in picolinamide and 163.89ppm in N-Chloropicolinamide. The carbonyl carbon is deshielded because of the -I effect of chlorine atom attached to the N- atom of NCP.Further, the compound has been confirmed by mass spectrum which shows parent ion peak at m/z: 156 and $M+2$ peak at 158. Formation of the peak at $(\mathrm{M}+2)$ also confirms the presence of chlorine in the molecule. The elemental analysis was in satisfactory agreement with the calculated values.

Calculated : C-45.86, H-3.19, N-17.58, O-10.43. Cl-22.97

Found : C-45.52, H-3.15, N-17.23, O-10.82, Cl-22.98

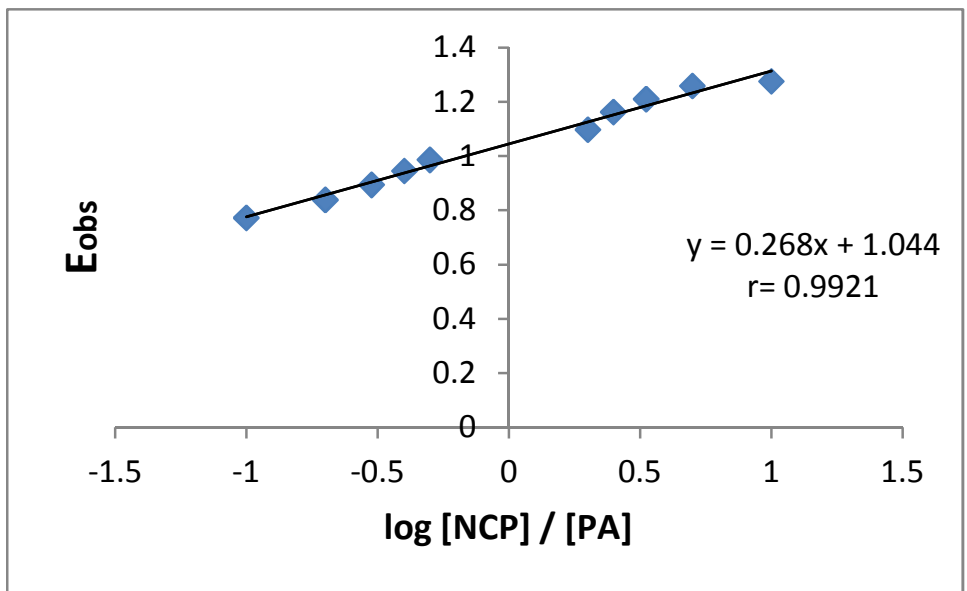

Fig.-2: To Determine the Value of Formal Redox Potential, a Graph of log [NCP]/[PA] Versus $E_{\text {obs }}$ is plotted. From the Intercept, the Formal Redox Potential is found to $1.04 \mathrm{~V}$.

NCP was found to be soluble in water, acetic acid, DMSO, DMF, sparingly soluble in ethanol, ethyl acetate, chloroform and insoluble in benzene, acetonitrile, dioxane and DCM. NCP stock solution can be prepared in $80 \%$ acetic acid and to prevent inter action with light the whole solution was kept in the amber-colored bottle. No appreciable change in concentration appearance and color indicating the fair degree of stability of the solution. The formal redox potential of NCP and PA couple is determined by a 
digital potentiometer with the platinum electrode and saturated aqueous calomel electrode. The formal redox potential of NCP/ PA couple was determined by measuring the potential in a mixture containing varying concentration ratios of $\mathrm{NCP}$ and $\mathrm{PA}$ in $80 \%$ acetic acid and $0.1 \mathrm{M} \mathrm{HCl}$. Since dilute solutions of NCP and PA were used, the is expected to be replaced by concentration terms in the Nernst equation,as-

$$
E_{o b s}=E^{0}+\frac{2.303 R T}{F}+\log \frac{[\mathrm{NCP}]}{[\mathrm{PA}]} .
$$

A plot of $\mathrm{E}_{\mathrm{obs}}$ against $\log [\mathrm{NCP}] /[\mathrm{PA}]$ from the above equation was made which yield a straight line with non-zero intercept (Fig-2) and the potential from it calculated to be $1.04 \mathrm{~V}$ at $25^{\circ} \mathrm{C}$. This value of formal redox potential of the NCP / PA couple viz. $\left(1.04 \mathrm{~V}\right.$ at $\left.25^{\circ} \mathrm{C}\right)$ is comparable to the redox potential value of $0.808 \mathrm{~V}$ for $\mathrm{N}$-Bromoisonicotinamide ${ }^{16}, 1.05 \mathrm{~V}$ for $\mathrm{N}^{-C h l o r o i s o n i c o t i n a m i d e}{ }^{17}, 1.02 \mathrm{~V}$ for $\mathrm{N}$ Chloronicotinamide ${ }^{18}, 1.16 \mathrm{~V}$ for bromamine $-\mathrm{T}^{19}, 1.14 \mathrm{~V}$ for chloramine $-\mathrm{T}^{20}, 1.18 \mathrm{~V}$ for $\mathrm{N}$ Chloropyrazinamide $^{21}$ and $0.797 \mathrm{~V}$ N-Bromonicotinamide. ${ }^{22}$

\section{REFERENCES}

1. M. Mathiyalagan, J. Indian Chem. Soc, 88, 865(2011).

2. E. S. Amis, J. Chem. Ed., 30, 351(1953).

3. V. Priya, M. Balasubramaniyan and M. Mathiyalagan, J. Chem. Pharm. Res., 3, 522(2011).

4. P. S. Ramakrishnan, Asian J. Chem., 12, 1096(2000).

5. Goyal and S. Kothari, J. Chem. Res., (S)2, 1259(2002).

6. Ashih Tomer and Arun kumar, J. Indian Chem. Soc., 83, 1153(2006).

7. J. Pusphalatha and K.Vivekanandan, J. Indian Chem. Soc., 86, 895 (2009).

8. S. Kavitha and I. Alphonse, Indian J. Chem., 44A, 715 (2005).

9. M. Mathiyalagan, V. Priya and J. John Basco, J. Indian Chem. Soc., 86, 453(2006).

10. N. A. Mohamed Farook, J. Solution chem, 36, 345(2007).

11. N. Mathiyalagan, K. Shenbagam and M. Kavitha, Asian J. Chem., 22, 265(2010).

12. D. V. Prabu, J. Indian Chem. Soc., 83(11), 1135(2007).

13. R. A. Singh, Kamini Singh, and S. K. Singh, J. Chem Pharm. Res., 2, 684(2010).

14. B. Ramkumar, Asian J. Chem., 13, 777(2001).

15. K. Senbagam and N. Mathiyalagan, Orient J. Chem., 29, 747(2013).

16. M. Balasubramaniyan and N. Mathiyalagan, Orient J. Chem., 26, 1541(2010).

17. V. Priya and N. Mathiyalagan, Asian J. Chem., 23, 1871(2011).

18. K. Vivekanandan and K, Nambi, Indian J. Chem., 35B, 1117(1996).

19. G. R. Nair, R. Lalithakumari and P. L. Senan, Talenta 525, 25(1978).

20. A. R. V. Murthy and B. S. Rao, Proc Indian Acad Sci., 69, 35(1952).

21. S. Parimala Vaijayanthi and N. Mathiyalagan, Inter J. Res. Inorganic Chem, 2, 13(2012).

22. L. Pushpalatha and K. Vivekanandan, J. Indian Chem. Soc., 84, 1119(2007).

[RJC-5213/2019] 